\title{
PROBABILISTIC-VALUED DECOMPOSABLE SET FUNCTIONS WITH RESPECT TO TRIANGLE FUNCTIONS
}

\author{
Lenka HALČINOVÁ, Ondrej HUTNÍK and Jana MOLNÁROVÁ⿴
}

\begin{abstract}
In the framework of generalized measure theory the probabilistic-valued decomposable set functions are introduced with triangle functions $\tau$ in an appropriate probabilistic metric space as natural candidates for the "addition", leading to the concept of $\tau$-decomposable measures. Several set functions, among them the classical (sub)measures, previously defined $\tau_{T}$-submeasures [11], $\tau_{L, A}$-submeasures $[8$ as well as recently introduced Shen's (sub)measures 23 are described and investigated in a unified way. Basic properties and characterizations of $\tau$-decomposable (sub)measures are also studied and numerous extensions of results from the above mentioned papers are provided.
\end{abstract}

\section{Introduction}

Real world applications often require dealing with such situations when the exact numerical values of (sub)measure of a set may not be provided, but at least some probabilistic assignment still could be done. A similar situation is discussed in the framework of information measures in 13. For instance, consider a grant agency providing a financial support for research in some area. From the set of all grant applications only "successful" (depending on some internal rules of agency) will receive certain amount of money. So, we have only a probabilistic information about measure of the set of "successful" grant applications. Of course, the knowledge of this information depends on many different aspects: total budget of money to be divided, internal rules of agency, quality of reviewers (if any), etc. Further examples are provided by lotteries, or guessing results when we have a probabilistic information about (counting) measure of possibilities to win the prize. A closely related concept can be found in Moore's interval mathematics [16, where the use of intervals in data processing is due to measurement inaccuracy and due to rounding. Here intervals can be considered in distribution function form linked to random variables uniformly distributed over the relevant intervals. These model examples resemble the original idea of Menger of PM-spaces, see [15, where the replacement of a positive number by a distance distribution function was motivated by thinking of situations where the exact distance between two objects may not be provided, but some probability assignment is still possible. Thus, the importance/diameter/measure of a set might be represented by a distance distribution function. Recently, probabilistic approaches were successfully applied to modelling uncertain logical arguments [10], to approximations of incomplete data [6], to inference rules playing an important role in non-monotonic reasoning [4, or to cluster structure ensemble [25].

In the paper by Hutník and Mesiar 11 the notion of $\tau_{T}$-submeasure was defined intended to be a certain (non-additive) set function $\gamma$ on a ring $\Sigma$ of subsets of a non-empty set $\Omega$ taking values in the set $\Delta^{+}$of distribution functions of non-negative random variables satisfying $\gamma_{\emptyset}=$ $\varepsilon_{0}$, "antimonotonicity" property $\gamma_{E} \geq \gamma_{F}$ whenever $E, F \in \Sigma$ with $E \subseteq F$, and "subadditivity" property of the form

$$
\gamma_{E \cup F}(x+y) \geq T\left(\gamma_{E}(x), \gamma_{F}(y)\right), \quad E, F \in \Sigma, x, y>0,
$$

with $T$ being a left-continuous t-norm. Here, $\varepsilon_{0}$ is the distribution function of Dirac random variable concentrated at point 0 . As it is shown in [11, such $\tau_{T}$-submeasures can be seen as fuzzy number-valued submeasures. In this case the value $\gamma_{E}$ may be seen as a non-negative LT-fuzzy number, see [3], where $\tau_{T}\left(\gamma_{E}, \gamma_{F}\right)$ corresponds to the $T$-sum of fuzzy numbers $\gamma_{E}$

\footnotetext{
${ }^{1}$ Mathematics Subject Classification (2010): Primary 54E70, Secondary 60A10, 60B05 Key words and phrases: Probabilistic metric space; decomposable measure; triangle function; triangular norm; aggregation function
} 
and $\gamma_{F}$. Also, each $\tau_{M}$-submeasure $\gamma$ with the minimum t-norm $M(x, y)=\min \{x, y\}$ can be represented by means of a non-decreasing system $\left(\eta_{\alpha}\right)_{\alpha \in[0,1]}$ of numerical submeasures as follows

$$
\gamma_{E}(x)=\sup \left\{\alpha \in[0,1] ; \eta_{\alpha}(E) \leq x\right\}, \quad E \in \Sigma .
$$

This representation resembles the horizontal representation $\left(S_{\alpha}\right)_{\alpha \in[0,1]}$ of a fuzzy subset $S$.

The study of probabilistic-valued set functions continued in papers [8] and [9], where a more general concept has been used. In fact, in [9] a generalization of $\tau_{T}$-submeasures was suggested which involves suitable operations $L$ replacing the standard addition + on $\overline{\mathbb{R}}_{+}$. On the other hand, since t-norms are rather special operations on the unit interval [0,1], the paper [8] deals with a number of possible generalizations based on aggregation functions in general studying certain properties of the corresponding probabilistic (sub)measures and their (sub)measure spaces.

Recently, in 23. Shen defined and studied a class of probabilistic (sub)measures which seem they do not fit to the concept of previously mentioned results of Hutník and Mesiar. In fact, Shen's definition of a probabilistic-valued $\top$-decomposable supmeasure $2 \mathfrak{M}: \Sigma \rightarrow \Delta^{+}$with the "subadditivity" property

$$
\mathfrak{M}_{E \cup F}(t) \geq \top\left(\mathfrak{M}_{E}(t), \mathfrak{M}_{F}(t)\right), \quad E, F \in \Sigma, t>0
$$

with $\top$ being a t-norm, corresponds to the notion of $\tau_{\max , T}$-submeasure defined in [9], however a deeper contextual understanding was still unclear from that paper. Thus, in this paper we provide a deep insight into all the mentioned notions being, in fact, special cases of a probabilistic-valued set function with respect to a triangle function. Recall that a triangle function $\tau$ is a binary operation on $\Delta^{+}$such that the triple $\left(\Delta^{+}, \tau, \leq\right)$ forms a commutative, partially ordered semigroup with neutral element $\varepsilon_{0}$.

More precisely, the notion of $\tau_{T}$-submeasure is related to the triangle function $\tau=\tau_{T}$ given by

$$
\tau_{T}(G, H)(x)=\sup _{u+v=x} T(G(u), H(v)), \quad G, H \in \Delta^{+}
$$

with $T$ being a left-continuous t-norm. Thus, the "subadditivity" property (1) resembles the "probabilistic analogue" of the triangle inequality in the Menger probabilistic metric space (under $T$ ), see [22]. Moreover, $\tau_{L, T}$-submeasures defined in [9, Definition 2.3] are related to the (triangle) function

$$
\tau_{L, T}(G, H)(x)=\sup _{L(u, v)=x} T(G(u), H(v)), \quad G, H \in \Delta^{+},
$$

with a suitable operation $L$ on $\overline{\mathbb{R}}_{+}$. Even more, Shen's considerations are related to the pointwisely defined (triangle) function

$$
\tau_{\top}(G, H)(t)=\top(G(t), H(t)), \quad G, H \in \Delta^{+},
$$

and the "subadditivity" property (2) is related to the triangle inequality of the corresponding probabilistic metric space. So, we can see that triangle functions are the main ingredient which connects all the mentioned notions of (sub)measure. Thus, considering a general triangle function $\tau$ on $\Delta^{+}$we define and study certain properties of $\tau$-decomposable (sub)measures on a ring $\Sigma$ of subsets of $\Omega \neq \emptyset$ in this general setting.

In the next section the short overview of basic notions and definitions is given. In Section 3 we introduce the basic object of our study: a $\tau$-decomposable set function with values in distance distribution functions and provide a number of concrete examples. Several properties of such set functions are then investigated in Section 4 and results related to probabilistic Hausdorff distance are provided in Section 5 generalizing the recent results of Shen [23].

\footnotetext{
${ }^{2}$ supmeasure in the terminology of Shen corresponds to submeasure in our terminology, see [23] Definition $4.1(\mathrm{v})]$
} 


\section{Basic notions and definitions}

In order to make the exposition self-contained, here we remind the reader the basic notions and constructions used in this paper.

Distribution functions Let $\Delta$ be the family of all distribution functions on the extended real line $\overline{\mathbb{R}}:=[-\infty,+\infty]$, i.e., $F: \overline{\mathbb{R}} \rightarrow[0,1]$ is non-decreasing, left continuous on the real line $\mathbb{R}$ with $F(-\infty)=0$ and $F(+\infty)=1$. A distance distribution function is a distribution function whose support is a subset of $\overline{\mathbb{R}}_{+}:=[0,+\infty]$, i.e., a distribution function $F: \overline{\mathbb{R}} \rightarrow[0,1]$ with $F(0)=0$. The class of all distance distribution functions will be denoted by $\Delta^{+}$.

For a distance distribution function $G$ and a non-negative constant $c \in \overline{\mathbb{R}}_{+}$we define the multiplication of $G$ by a constant $c$ as follows

$$
(c \odot G)(x):= \begin{cases}\varepsilon_{0}(x), & c \in\{0,+\infty\}, \\ G\left(\frac{x}{c}\right), & \text { otherwise. }\end{cases}
$$

Clearly, $c \odot G \in \Delta^{+}$.

A triangle function is a mapping $\tau: \Delta^{+} \times \Delta^{+} \rightarrow \Delta^{+}$which is symmetric, associative, non-decreasing in each variable and has $\varepsilon_{0}$ as the identity, where $\varepsilon_{0}$ is the distribution function of Dirac random variable concentrated at point 0 . More precisely, for $a \in[-\infty,+\infty$ [ we put

$$
\varepsilon_{a}(x):= \begin{cases}1 & \text { for } x>a \\ 0 & \text { otherwise }\end{cases}
$$

For more details on triangle functions we recommend the overview paper [20]. The most important triangle functions are those obtained from certain aggregation functions, especially t-norms.

Aggregation functions A binary aggregation function $A:[0,1]^{2} \rightarrow[0,1]$ is a non-decreasing function in both components with the boundary conditions $A(0,0)=0$ and $A(1,1)=1$. The class of all binary aggregation functions will be denoted by $\mathscr{A}$. For more details on aggregation functions we recommend the monograph 5 .

A triangular norm, shortly a t-norm, is a commutative lattice ordered semi-group on [0,1] with identity 1 . The most important are the minimum t-norm $M(x, y):=\min \{x, y\}$, the product t-norm $\Pi(x, y):=x y$, the Łukasiewicz t-norm $W(x, y):=\max \{x+y-1,0\}$, and the drastic product t-norm

$$
D(x, y):= \begin{cases}\min \{x, y\} & \text { for } \max \{x, y\}=1 \\ 0 & \text { otherwise }\end{cases}
$$

For more information about t-norms and their properties we refer to books [14, 22]. Throughout this paper $\mathscr{T}$ denotes the class of all t-norms.

Binary operations on non-negative reals Let us denote by $\mathscr{L}$ the set of all binary operations $L$ on $\overline{\mathbb{R}}_{+}$such that

(i) $L$ is commutative and associative;

(ii) $L$ is jointly strictly increasing, i.e., for all $u_{1}, u_{2}, v_{1}, v_{2} \in \overline{\mathbb{R}}_{+}$with $u_{1}<u_{2}, v_{1}<v_{2}$ holds $L\left(u_{1}, v_{1}\right)<L\left(u_{2}, v_{2}\right)$

(iii) $L$ is continuous on $\overline{\mathbb{R}}_{+} \times \overline{\mathbb{R}}_{+}$;

(iv) $L$ has 0 as its neutral element. 
Observe that $L \in \mathscr{L}$ is a jointly increasing pseudo-addition on $\overline{\mathbb{R}}_{+}$in the sense of [24]. The usual (class of) examples of operations in $\mathscr{L}$ are

$$
\begin{aligned}
K_{\alpha}(x, y) & :=\left(x^{\alpha}+y^{\alpha}\right)^{\frac{1}{\alpha}}, \quad \alpha>0, \\
K_{\infty}(x, y) & :=\max \{x, y\} .
\end{aligned}
$$

In general, $L \in \mathscr{L}$ if and only if there is a (possibly empty) system (]$a_{k}, b_{k}[)_{k \in K}$ of pairwise disjoint open subintervals of $] 0,+\infty\left[\right.$, and a system $\left(\ell_{k}\right)_{k \in K}$ of increasing bijections $\ell_{k}:\left[a_{k}, b_{k}\right] \rightarrow \overline{\mathbb{R}}_{+}$so that

$$
L(x, y)= \begin{cases}\ell_{k}^{-1}\left(\ell_{k}(x)+\ell_{k}(y)\right) & \text { if }(x, y) \in] a_{k}, b_{k}\left[^{2},\right. \\ \max \{x, y\} & \text { otherwise. }\end{cases}
$$

For more details see [14. Further, for $(L, A) \in \mathscr{L} \times \mathscr{A}$ define the function

$$
\tau_{L, A}(G, H)(x):=\sup _{L(u, v)=x} A(G(u), H(v)), \quad G, H \in \Delta^{+} .
$$

However, $\tau_{L, A}$ need not be associative in general, thus need not be a triangle function, but it has good properties on $\Delta^{+}$. As it is shown in [20, the appropriate choice for $A$ is a semicopula $S$ - a binary aggregation function on $[0,1]$ with 1 as its neutral element. Indeed, the left-continuity of $S$ guarantees that $\tau_{L, S}$ is a binary operation on $\Delta^{+}$, cf. [20, Lemma 7.1]. Also, for any semi-copula $S$,

$$
\tau_{K_{\infty}, S}(G, H)(x)=S(G(x), H(x))
$$

is the operation pointwisely induced by $S$ on $\Delta^{+}$. For more information about (triangular) functions in connection with various aggregation functions and their properties we refer to [20].

Probabilistic metric spaces The function (6), at least for a special choice of $L$ and $A$, naturally arises in the context of probabilistic metric spaces. Recall that a probabilistic metric space (PM-space, for short) is a non-empty set $\Omega$ together with a family $\mathscr{F}$ of probability functions $F_{p, q}(x)$ (interpreted as the probability that distance between elements $p, q$ of $\Omega$ is less than $x$ ) with $F_{p, q}(0)=0$ satisfying

(i) $F_{p, q}=\varepsilon_{0}$ if and only if $p=q$;

(ii) $F_{p, q}=F_{q, p}$;

(iii) $F_{p, r} \geq \tau\left(F_{p, q}, F_{q, r}\right)$,

where $\tau$ is a triangle function on $\Delta^{+}$. A particular case includes the triangle inequality

$$
F_{p, r}(L(x, y)) \geq A\left(F_{p, q}(x), F_{q, r}(y)\right)
$$

which holds for all $p, q, r \in \Omega$ and all real $x, y$ with a suitable binary aggregation function $A$ and operation $L$. In particular, the triple $\left(\Omega, \mathscr{F}, \tau_{L, T}\right)$ with $\tau_{L, T}$ given by (6) for $A=T$ (a left-continuous t-norm) is called an L-Menger PM-space (under $T$ ). For $L=K_{\infty}$ we get a non-Archimedean Menger PM-space $\left(\Omega, \mathscr{F}, \tau_{K_{\infty}, T}\right)$ with $T \in \mathscr{T}$. Note that if $\mathscr{F}$ satisfies $F_{p, p}=\varepsilon_{0}$ for each $p \in \Omega$ and properties (ii) and (iii), then the triple $(\Omega, \mathscr{F}, \tau)$ will be called a probabilistic pseudo-metric space (PpM-space, for short). In general, different triangle functions lead to PM-spaces with different geometric and topological properties.

\section{Probabilistic-valued decomposable set functions w.r.t. a triangle function}

As we have already mentioned in the introduction, a natural origin of probabilistic-valued set functions comes from the fact that they work in such situations in which we have only a 
probabilistic information about measure of a set. For example, if rounding of reals is considered, then the uniform distributions over intervals describe our information about the measure of a set. Thus, the probabilistic-valued (sub)measures represent the concept of (sub)measures probabilistically rather than deterministically.

Here we introduce the basic notion of probabilistic decomposable (sub)measure in its general form. For better readability we also use the following conventions:

(i) for a probabilistic-valued set function $\gamma: \Sigma \rightarrow \Delta^{+}$we write $\gamma_{E}(x)$ instead of $\gamma(E)(x)$;

(ii) since $\Delta^{+}$is the set of all distribution functions with support $\overline{\mathbb{R}}_{+}$, we state the expression for a mapping $\gamma: \Sigma \rightarrow \Delta^{+}$just for positive values of $x$. In case $x \leq 0$ we always suppose $\gamma \cdot(x)=0$.

Definition 3.1. Let $\tau$ be a triangle function on $\Delta^{+}$and $\Sigma$ be a ring of subsets of $\Omega \neq \emptyset$. A mapping $\gamma: \Sigma \rightarrow \Delta^{+}$with $\gamma_{\emptyset}=\varepsilon_{0}$ is said to be a $\tau$-decomposable submeasure, if $\gamma_{E \cup F} \geq$ $\tau\left(\gamma_{E}, \gamma_{F}\right)$ for each disjoint sets $E, F \in \Sigma$. If in the preceding inequality equality holds, then $\gamma$ is said to be a $\tau$-decomposable measure on $\Sigma$.

Remark 3.2. In fact, a mapping $\gamma: \Sigma \rightarrow \Delta^{+}$is a probabilistic-valued set function, where the value of $\gamma_{E}$ at $x$ may be interpreted as the probability that a numerical (sub)measure of the set $E$ is less than $x$. A triangle function $\tau$ is a natural choice for "aggregation" of $\gamma_{E}$ and $\gamma_{F}$ in order to compare them with $\gamma_{E \cup F}$. For a $\tau$-decomposable measure $\gamma$ we naturally expect that $\gamma_{E \cup F}$ is the same distance distribution function as $\gamma_{F \cup E}$ for disjoint sets $E, F \in \Sigma$, from which follows that $\tau\left(\gamma_{E}, \gamma_{F}\right)=\tau\left(\gamma_{F}, \gamma_{E}\right)$, i.e., $\tau$ has to be commutative. Moreover, from the natural equality $\gamma_{(E \cup F) \cup G}=\gamma_{E \cup(F \cup G)}$ we obtain $\tau\left(\tau\left(\gamma_{E}, \gamma_{F}\right), \gamma_{G}\right)=\tau\left(\gamma_{E}, \tau\left(\gamma_{F}, \gamma_{G}\right)\right)$, i.e., $\tau$ has to be associative. Since $\gamma_{E}=\gamma_{E \cup \emptyset}=\tau\left(\gamma_{E}, \gamma_{\emptyset}\right)=\tau\left(\gamma_{E}, \varepsilon_{0}\right)$, then $\varepsilon_{0}$ has to be neutral element of $\tau$. The role of monotonicity of $\tau$ (a non-decreasing function in each place) will be examined in what follows.

Theorem 3.3. Let $\tau$ be a triangle function on $\Delta^{+}$and $\Sigma$ be a ring of subsets of $\Omega \neq \emptyset$. Then each $\tau$-decomposable measure $\gamma$ is "antimonotone" on $\Sigma$, i.e., $\gamma_{E} \geq \gamma_{F}$ whenever $E, F \in \Sigma$ such that $E \subseteq F$.

Proof. From monotonicity of triangular function $\tau$ with $\varepsilon_{0}$ as identity we easily have

$$
\gamma_{F}=\gamma_{E \cup(F \backslash E)}=\tau\left(\gamma_{E}, \gamma_{F \backslash E}\right) \leq \tau\left(\gamma_{E}, \varepsilon_{0}\right)=\gamma_{E}
$$

whenever $E, F \in \Sigma, E \subseteq F$.

Remark 3.4. The probabilistic interpretation of this property is as follows: the probability that a numerical measure of the set $E$ is less than $x$ is greater than or equal to the probability that a numerical measure of the set $F$ is less than $x$.

"Antimonotonicity" property does not hold for arbitrary $\tau$-decomposable submeasures $\gamma$ : $\Sigma \rightarrow \Delta^{+}$. Therefore, in [8, Definition 2.1] we have considered the notion of probabilistic submeasure w.r.t. a function $\tau=\tau_{L, A}$ being a $\tau_{L, A}$-decomposable antimonotone submeasure on $\Sigma$ where the "subadditivity" property $\gamma_{E \cup F} \geq \tau_{L, A}\left(\gamma_{E}, \gamma_{F}\right)$ holds for arbitrary sets $E, F \in \Sigma$. Therefore we state the following easy observation.

Theorem 3.5. Let $\tau$ be a triangle function on $\Delta^{+}$and $\Sigma$ be a ring of subsets of $\Omega \neq \emptyset$. If $\gamma$ : $\Sigma \rightarrow \Delta^{+}$is a $\tau$-decomposable antimonotone submeasure, then the inequality $\gamma_{E \cup F} \geq \tau\left(\gamma_{E}, \gamma_{F}\right)$ holds for arbitrary sets $E, F \in \Sigma$.

Proof. For $E \cap F=\emptyset$ the inequality holds by Definition 3.1 Let $E, F \in \Sigma$ and $E \cap F \neq \emptyset$. Then the inequality

$$
\gamma_{E \cup F}=\gamma_{E \cup((E \cup F) \backslash E)} \geq \tau\left(\gamma_{E}, \gamma_{(E \cup F) \backslash E}\right) \geq \tau\left(\gamma_{E}, \gamma_{F}\right)
$$

follows from antimonotonicity of $\gamma$ and monotonicity of triangle functions. 
Now we are in position to provide some examples of $\tau$-decomposable measures based on various constructions and related to different classes of aggregation functions appearing in the definition of the underlying triangle function. Note that under the "numerical (sub)measure" we mean a real-valued set function with the (usual) (sub)additivity property.

Example 3.6. Let $m$ be a numerical (additive) measure on $\Sigma$. If $m$ is $L$-decomposable, i.e., $m(E \cup F)=L(m(E), m(F))$ with $L \in \mathscr{L}$ and disjoint sets $E, F \in \Sigma$, then for any $\Phi \in \Delta^{+}$ the set function $\gamma^{\Phi}: \Sigma \rightarrow \Delta^{+}$defined by $\gamma_{E}^{\Phi}:=m(E) \odot \Phi$ is a $\tau_{L, M}$-decomposable measure on $\Sigma$, where $\tau_{L, M}$ is given by (4) with the minimum t-norm $T=M$. Indeed, "additivity" property follows from [22, Section 7.7]: the function $\tau_{L, M}$ is the only triangular function with the property

$$
\tau_{L, M}\left(c_{1} \odot H, c_{2} \odot H\right)=L\left(c_{1}, c_{2}\right) \odot H, \quad c_{1}, c_{2} \in \overline{\mathbb{R}}_{+}, H \in \Delta^{+} .
$$

Notice that $\gamma^{\Phi}$ need not be a $\tau_{L, T}$-decomposable measure for $T \neq M$.

Example 3.7. Shen's T-probabilistic decomposable measures, cf. [23]: this class of measures $\mathfrak{M}: \Sigma \rightarrow \Delta^{+}$of the form $\mathfrak{M}_{E \cup F}(t)=\top\left(\mathfrak{M}_{E}(t), \mathfrak{M}_{F}(t)\right)$ for disjoint $E, F \in \Sigma$ corresponds to the class of $\tau$-decomposable measures w.r.t. the triangle function $\Pi_{\top}: \Delta^{+} \times \Delta^{+} \rightarrow \Delta^{+}$of the form

$$
\Pi_{\top}(G, H)(t)=\top(G(t), H(t)), \quad G, H \in \Delta^{+},
$$

with $\top$ being a t-norm, or, equivalently, to a $\tau_{\max , T}$-decomposable measure. According to [20, Theorem 5.2] left-continuity of t-norm $\top$ is a necessary and sufficient condition for $\Pi_{\top}$ being a triangle function. Note that Shen does not consider the left-continuity of $T$ in his definition of a T-probabilistic decomposable measure, cf. [23, Definition 4.1]. Moreover, Shen's considerations are made on a $\sigma$-algebra instead of a ring of subsets of $\Omega \neq \emptyset$ to provide a countable extension of $T$-decomposable measures.

Example 3.8. Convolution of distance distribution functions is a binary operation $*$ on $\Delta^{+}$ given by

$$
\tau_{*}(G, H)(x):=(G * H)(x)= \begin{cases}0, & x=0, \\ \int_{0}^{x} G(x-t) \mathrm{d} H(t), & x \in] 0,+\infty[, \\ 1, & x=+\infty,\end{cases}
$$

for each $G, H \in \Delta^{+}$, where the integral is meant in the sense of Lebesgue-Stieltjes. According to [20, Theorem 13.4 and Definition 13.1] $\tau_{*}$ is a triangle function on $\Delta^{+}$. The corresponding $\tau_{*}$-decomposable (sub)measure $\gamma$ provides an extension of a notion of (sub)measure to (sub)measures which can be used in the Wald spaces - those involving the convolution $*$ of distance distribution functions, but also in a wider class of PM-spaces.

If $m$ is a numerical measure on $\Sigma$, then the set function $\gamma: \Sigma \rightarrow \Delta^{+}$given by $\gamma_{E}=\varepsilon_{m(E)}$ for $E \in \Sigma$ is a $\tau_{*}$-decomposable measure, because

$$
\gamma_{E \cup F}=\varepsilon_{m(E \cup F)}=\varepsilon_{m(E)+m(F)}=\tau_{*}\left(\varepsilon_{m(E)}, \varepsilon_{m(F)}\right)=\tau_{*}\left(\gamma_{E}, \gamma_{F}\right),
$$

\section{cf. [20, Theorem $14.1(\mathrm{f})]$.}

The latter example of mapping $\gamma_{E}=\varepsilon_{m(E)}$ is also interesting from the viewpoint of other triangle functions. It gives rise claiming that each numerical (additive) measure can be regarded as a probabilistic-valued decomposable measure. Thus, $\tau$-decomposable measures are extensions of the classical measure.

Example 3.9. Let $\mu: \Sigma \rightarrow[0,+\infty)$ be a set function with $\mu(\emptyset)=0$ and for $E \in \Sigma$ put $\gamma_{E}=\varepsilon_{\mu(E)}$. Due to [20, Theorem 14.1] we have the following results:

(i) if $\mu$ is additive, then $\gamma$ is a $\tau_{D}$-decomposable measure;

(ii) if $\mu$ is additive and $T \in \mathscr{T}$ is continuous, then $\gamma$ is a $\tau_{T}$-decomposable measure; 
(iii) if $\mu$ is $L$-decomposable with $L \in \mathscr{L}$, and $T \in \mathscr{T}$ is continuous, then $\gamma$ is a $\tau_{L, T^{-}}$ decomposable measure;

(iv) if $\mu$ is $L$-decomposable with $L \in \mathscr{L}$ and $Q$ is a symmetric quasi-copula with an associative dual quasi-copula $\bar{Q}$, then $\gamma$ is a $\rho_{L, Q}$-decomposable measure, where

$$
\rho_{L, Q}(G, H)(x)=\inf _{L(u, v)=x} \bar{Q}(G(u), H(v)), \quad G, H \in \Delta^{+} ;
$$

(v) if $\mu$ is $K_{\infty}$-decomposable, then $\gamma$ is $\Pi_{\top}$-decomposable measure for each $\top \in \mathscr{T}$, where $\Pi_{\top}$ is given by (8), i.e., $\gamma$ is a Shen's $\top$-probabilistic decomposable measure. Note that if $\mu$ is additive, then $\gamma$ need not be a $\Pi_{\top}$-decomposable measure. For instance, considering $\top=M$ (the minimum t-norm) yields

$$
\varepsilon_{\mu(E)+\mu(F)}(x) \neq M\left(\varepsilon_{\mu(E)}(x), \varepsilon_{\mu(F)}(x)\right)
$$

whenever $\mu(E) \neq \mu(F) \neq \emptyset$.

\section{Properties and constructions of decomposable (sub)measures}

Clearly, each $\tau$-decomposable measure on $\Sigma$ is a $\tau$-decomposable submeasure on $\Sigma$. The following result provides a characterization of the class of $\tau$-decomposable measures on $\Sigma$. In fact, it provides a generalization of [23, Theorem 4.1] to an arbitrary triangle function.

Theorem 4.1. Let $\tau$ be a triangle function on $\Delta^{+}$. Then $\gamma$ is a $\tau$-decomposable measure on $\Sigma$ if and only if $\tau\left(\gamma_{E \cup F}, \gamma_{E \cap F}\right)=\tau\left(\gamma_{E}, \gamma_{F}\right)$ for each $E, F \in \Sigma$.

Proof. " $\Leftarrow "$ If $E \cap F=\emptyset$, then $\gamma_{E \cap F}=\varepsilon_{0}$ and

$$
\tau\left(\gamma_{E}, \gamma_{F}\right)=\tau\left(\gamma_{E \cup F}, \gamma_{E \cap F}\right)=\tau\left(\gamma_{E \cup F}, \varepsilon_{0}\right)=\gamma_{E \cup F}
$$

$" \Rightarrow$ " Since $E \cup F=(E \cap F) \cup(E \backslash F) \cup(F \backslash E)$, then we have

$$
\begin{aligned}
\tau\left(\gamma_{E \cap F}, \gamma_{E \cup F}\right) & =\tau\left(\gamma_{E \cap F}, \tau\left(\gamma_{E \cap F}, \tau\left(\gamma_{E \backslash F}, \gamma_{F \backslash E}\right)\right)=\tau\left(\gamma_{E \cap F}, \tau\left(\tau\left(\gamma_{E \cap F}, \gamma_{E \backslash F}\right), \gamma_{F \backslash E}\right)\right)\right. \\
& =\tau\left(\gamma_{E \cap F}, \tau\left(\gamma_{E}, \gamma_{F \backslash E}\right)\right)=\tau\left(\gamma_{E \cap F}, \tau\left(\gamma_{E}, \gamma_{F \backslash E}\right)\right)=\tau\left(\gamma_{E}, \tau\left(\gamma_{E \cap F}, \gamma_{F \backslash E}\right)\right) \\
& =\tau\left(\gamma_{E}, \gamma_{F}\right)
\end{aligned}
$$

which completes the proof.

Now we are interested in a question to generate new decomposable (sub)measures from given ones. For that reason we will need the following notion, cf. [21, Definition 6.1]: Let $(X, \leq)$ be a partially ordered set and let $f$ and $g$ be two binary operations on $X$. Then $f$ dominates $g$, written $f \gg g$, if, for all $x, y, u, v \in X$,

$$
f(g(x, y), g(u, v)) \geq g(f(x, u), f(y, v)) .
$$

It is well known that dominance is an antisymmetric and reflexive, but not transitive relation on the set of triangle functions, cf. [21, Corollary 6.1]. Note that the idea of dominance appears in much previous paper [19] in the context of aggregation functions, or even in [1] in the context of triangular norms.

A triangle function $\tau$, such that for each $c \in \overline{\mathbb{R}}_{+}$and each $G, H \in \Delta^{+}$it holds

$$
c \odot \tau(G, H)=\tau(c \odot G, c \odot H)
$$

will be called a distributive triangle function. Recall that the operation $\odot$ is defined by (5). The standard examples of distributive triangle functions are $\tau_{T}$ and $\Pi_{\top}$.

Theorem 4.2. Let $\tau, \vartheta$ be two triangle functions on $\Delta^{+}$and $\gamma^{1}, \gamma^{2}: \Sigma \rightarrow \Delta^{+}$be $\tau$-decomposable measures. Then 
(i) if $\tau$ is distributive, the set function $\gamma:=c \odot \gamma^{1}$ is a $\tau$-decomposable measure for each $c \in \overline{\mathbb{R}}_{+}$;

(ii) the set function $\zeta:=\tau\left(\gamma^{1}, \gamma^{2}\right)$ is a $\tau$-decomposable measure;

(iii) the set function $\lambda:=\vartheta\left(\gamma^{1}, \gamma^{2}\right)$ is a $\tau$-decomposable submeasure if and only if $\vartheta \gg \tau$.

Proof. Consider $E, F \in \Sigma$ such that $E \cap F=\emptyset$. It is easy to verify that $\gamma_{\emptyset}=\zeta_{\emptyset}=\varepsilon_{0}$.

(i) Immediately, by distributivity of $\tau$ we get

$$
\gamma_{E \cup F}=c \odot \gamma_{E \cup F}^{1}=c \odot \tau\left(\gamma_{E}^{1}, \gamma_{F}^{1}\right)=\tau\left(c \odot \gamma_{E}^{1}, c \odot \gamma_{F}^{1}\right)=\tau\left(\gamma_{E}, \gamma_{F}\right) .
$$

(ii) It follows from associativity of triangle functions that

$$
\begin{aligned}
\zeta_{E \cup F} & =\tau\left(\gamma_{E \cup F}^{1}, \gamma_{E \cup F}^{2}\right)=\tau\left(\tau\left(\gamma_{E}^{1}, \gamma_{F}^{1}\right), \gamma_{E \cup F}^{2}\right)=\tau\left(\gamma_{E}^{1}, \tau\left(\gamma_{E \cup F}^{2}, \gamma_{F}^{1}\right)\right)=\tau\left(\gamma_{E}^{1}, \tau\left(\tau\left(\gamma_{E}^{2}, \gamma_{F}^{2}\right), \gamma_{F}^{1}\right)\right) \\
& \left.=\tau\left(\gamma_{E}^{1}, \tau\left(\gamma_{E}^{2}, \tau\left(\gamma_{F}^{2}, \gamma_{F}^{1}\right)\right)\right)=\tau\left(\tau\left(\gamma_{E}^{1}, \gamma_{E}^{2}\right), \tau\left(\gamma_{F}^{1}, \gamma_{F}^{2}\right)\right)\right)=\tau\left(\zeta_{E}, \zeta_{F}\right) .
\end{aligned}
$$

(iii) If $\lambda:=\vartheta\left(\gamma^{1}, \gamma^{2}\right)$ is a $\tau$-decomposable submeasure, then $\lambda_{E \cup F} \geq \tau\left(\lambda_{E}, \lambda_{F}\right)$, i.e.,

$$
\vartheta\left(\gamma_{E \cup F}^{1}, \gamma_{E \cup F}^{2}\right) \geq \tau\left(\vartheta\left(\gamma_{E}^{1}, \gamma_{E}^{2}\right), \vartheta\left(\gamma_{F}^{1}, \gamma_{F}^{2}\right)\right)
$$

Since

therefore

$$
\vartheta\left(\gamma_{E \cup F}^{1}, \gamma_{E \cup F}^{2}\right)=\vartheta\left(\tau\left(\gamma_{E}^{1}, \gamma_{F}^{1}\right), \tau\left(\gamma_{E}^{2}, \gamma_{F}^{2}\right)\right)
$$

$$
\vartheta\left(\tau\left(\gamma_{E}^{1}, \gamma_{F}^{1}\right), \tau\left(\gamma_{E}^{2}, \gamma_{F}^{2}\right)\right) \geq \tau\left(\vartheta\left(\gamma_{E}^{1}, \gamma_{E}^{2}\right), \vartheta\left(\gamma_{F}^{1}, \gamma_{F}^{2}\right)\right)
$$

which means that $\vartheta \gg \tau$.

On the other hand, if $\vartheta \gg \tau$, then the inequality (9) holds and the equalities

$$
\begin{aligned}
& \vartheta\left(\tau\left(\gamma_{E}^{1}, \gamma_{F}^{1}\right), \tau\left(\gamma_{E}^{2}, \gamma_{F}^{2}\right)\right)=\vartheta\left(\gamma_{E \cup F}^{1}, \gamma_{E \cup F}^{2}\right)=\lambda_{E \cup F}, \\
& \tau\left(\vartheta\left(\gamma_{E}^{1}, \gamma_{E}^{2}\right), \vartheta\left(\gamma_{F}^{1}, \gamma_{F}^{2}\right)\right)=\tau\left(\lambda_{E}, \lambda_{F}\right)
\end{aligned}
$$

imply the result.

As we can see, a $\tau$-sum of two $\tau$-decomposable measures produces a $\tau$-decomposable measure. The same is true for (antimonotone) $\tau$-decomposable submeasures. Replacing a triangle function by a more general aggregation operator (on $\Delta^{+}$) may also produce a desired set function.

Recall that an ( $n$-ary) aggregation operator on $\Delta^{+}$is a mapping $\alpha:\left(\Delta^{+}\right)^{n} \rightarrow \Delta^{+}$which is non-decreasing in each place with the boundary condition $\alpha\left(\varepsilon_{0}, \ldots, \varepsilon_{0}\right)=\varepsilon_{0}$. Also, for domination between aggregation operators we refer to [19]: we say that an ( $n$-ary) aggregation operator $\alpha$ dominates an (m-ary) aggregation operator $\beta$, we write $\alpha \gg \beta$, if for all $H_{i, j} \in \Delta^{+}$ with $i \in\{1, \ldots, m\}$ and $j \in\{1, \ldots, n\}$, the following property holds:

$$
\alpha\left(\beta\left(H_{1,1}, \ldots, H_{m, 1}\right), \ldots, \beta\left(H_{1, n}, \ldots H_{m, n}\right)\right) \geq \beta\left(\alpha\left(H_{1,1}, \ldots, H_{1, n}\right), \ldots, \alpha\left(H_{m, 1}, \ldots, H_{m, n}\right)\right) .
$$

Theorem 4.3. Let $\tau_{i}$ be triangle functions on $\Delta^{+}$and $\gamma^{i}: \Sigma \rightarrow \Delta^{+}$be $\tau_{i}$-decomposable submeasures for $i=1, \ldots, n, n \in \mathbb{N}$. If $\tau$ is a triangle function on $\Delta^{+}$such that $\tau \leq \tau_{i}$ for each $i=1, \ldots, n$, and $\alpha$ is an (n-ary) aggregation operator on $\Delta^{+}$such that $\alpha \gg \tau$, then $\gamma:=\alpha\left(\gamma^{1}, \cdots \gamma^{n}\right)$ is a $\tau$-decomposable submeasure on $\Sigma$.

Proof. Immediately, for each $E, F \in \Sigma$ we have

$$
\begin{aligned}
\gamma_{E \cup F} & =\alpha\left(\gamma_{E \cup F}^{1}, \ldots, \gamma_{E \cup F}^{n}\right) \geq \alpha\left(\tau_{1}\left(\gamma_{E}^{1}, \gamma_{F}^{1}\right), \ldots, \tau_{n}\left(\gamma_{E}^{n}, \gamma_{F}^{n}\right)\right) \\
& \geq \alpha\left(\tau\left(\gamma_{E}^{1}, \gamma_{F}^{1}\right), \ldots, \tau\left(\gamma_{E}^{n}, \gamma_{F}^{n}\right)\right) \geq \tau\left(\alpha\left(\gamma_{E}^{1}, \cdots, \gamma_{E}^{n}\right), \alpha\left(\gamma_{F}^{1}, \cdots, \gamma_{F}^{n}\right)\right) \\
& =\tau\left(\gamma_{E}, \gamma_{F}\right) .
\end{aligned}
$$

Finally, by boundary condition we get $\gamma_{\emptyset}=\alpha\left(\gamma_{\emptyset}^{1}, \ldots, \gamma_{\emptyset}^{n}\right)=\alpha\left(\varepsilon_{0}, \ldots, \varepsilon_{0}\right)=\varepsilon_{0}$. 
Remark 4.4. Especially, it is well-known that the arithmetic mean $A M$ on $[0,1]$ dominates $W$, cf. [18, thus $\Pi_{A M}$ dominates $\tau_{L, W}$ for an arbitrary $L \in \mathscr{L}$, cf. 21. So, $\Pi_{A M}$-aggregation of $\tau_{L, W}$-decomposable submeasures, i.e., a set function $\gamma:=\Pi_{A M}\left(\gamma^{1}, \ldots, \gamma^{n}\right)$, is again a $\tau_{L, W^{-}}$ decomposable submeasure.

The following construction is based on the observation taken from a construction of product PM-spaces for the case of finite products, cf. [21]. For more details about pseudo-metrics generated by probabilistic-valued decomposable (sub)measures see below.

Theorem 4.5. Let $\tau_{i}$ be a (finite) family of triangle functions on $\Delta^{+}$and $\gamma^{i}: \Sigma_{i} \rightarrow \Delta^{+}$be $\tau_{i}$-decomposable antimonotone submeasures for $i=1, \ldots n, n \in \mathbb{N}$. Let $\left(\Sigma_{i}, \rho^{i}, \tau_{i}\right)$ be a family of PpM-spaces with $\rho_{E_{i}, F_{i}}^{i}:=\gamma_{E_{i} \triangle F_{i}}^{i}$, and $\alpha$ be an (n-ary) aggregation operator on $\Delta^{+}$. If there exists a triangle function $\tau$ on $\Delta^{+}$such that $\alpha \gg \tau$ and that $\tau \leq \tau_{i}$ for every $i=1, \ldots, n$, then the triple $(\Sigma, \rho, \tau)$ is a PpM-space, where $\rho$ is defined on $\Sigma:=\prod_{i=1}^{n} \Sigma_{i}$ by

$$
\rho_{E, F}:=\alpha\left(\rho_{E_{1}, F_{1}}^{1}, \ldots, \rho_{E_{n}, F_{n}}^{n}\right)
$$

Proof. We prove that $\rho$ is a pseudo-metric on $\Sigma$ w.r.t. $\tau$. Clearly, $\rho_{E, E}=\varepsilon_{0}$ for each $E \in \Sigma$ and $\rho_{E, F}=\rho_{F, E}$ for each $E, F \in \Sigma$. Taking $E, F, G \in \Sigma$ we obtain

$$
\begin{aligned}
\tau\left(\rho_{E, F}, \rho_{F, G}\right) & =\tau\left(\alpha\left(\rho_{E_{1}, F_{1}}^{1}, \ldots, \rho_{E_{n}, F_{n}}^{n}\right), \alpha\left(\rho_{F_{1}, G_{1}}^{1}, \ldots, \rho_{F_{n}, G_{n}}^{n}\right)\right) \\
& \leq \alpha\left(\tau\left(\rho_{E_{1}, F_{1}}^{1}, \rho_{F_{1}, G_{1}}^{1}\right), \ldots, \tau\left(\rho_{E_{n}, F_{n}}^{n}, \rho_{F_{n}, G_{n}}^{n}\right)\right) \\
& \leq \alpha\left(\tau_{1}\left(\rho_{E_{1}, F_{1}}^{1}, \rho_{F_{1}, G_{1}}^{1}\right), \ldots, \tau_{n}\left(\rho_{E_{n}, F_{n}}^{n}, \rho_{F_{n}, G_{n}}^{n}\right)\right) \\
& \leq \alpha\left(\rho_{E_{1}, G_{1}}^{1}, \ldots, \rho_{E_{n}, G_{n}}^{n}\right)=\rho_{E, G},
\end{aligned}
$$

which completes the proof.

We now extend results from [9] to a general case of $\tau$-decomposable (sub)measures instead of $\tau_{L, T}$-submeasures considered therein.

Theorem 4.6. Let $\tau$ be a triangle function on $\Delta^{+}$and $\gamma: \Sigma \rightarrow \Delta^{+}$be a $\tau$-decomposable antimonotone submeasure. Define the mapping $\rho: \Sigma \times \Sigma \rightarrow \Delta^{+}$by the formula $\rho_{E, F}:=\gamma_{E \triangle F}$ with $E, F \in \Sigma$. Then the triple $(\Sigma, \rho, \tau)$ is a PpM-space (under $\tau$ ).

Proof. Clearly, $\rho_{E, E}=\gamma_{E \triangle E}=\varepsilon_{0}$ for each $E \in \Sigma$. Similarly, symmetry is trivial. It is enough to prove the triangle inequality. By Theorem 3.5 consider arbitrary sets $E, F, G \in \Sigma$. Since

$$
E \triangle G \subset(E \triangle F) \cup[(G \backslash(E \cup F)) \cup((E \cap F) \backslash G)],
$$

and

$$
(G \backslash(E \cup F)) \cup((E \cap F) \backslash G) \subset F \triangle G,
$$

then antimonotonicity of $\gamma$ yields

$$
\begin{aligned}
\rho_{E, G}=\gamma_{E \triangle G} & \geq \gamma_{(E \triangle F) \cup[(G \backslash(E \cup F)) \cup((E \cap F) \backslash G)]} \geq \tau\left(\gamma_{E \triangle F}, \gamma_{(G \backslash(E \cup F)) \cup((E \cap F) \backslash G)}\right) \\
& \geq \tau\left(\gamma_{E \triangle F}, \gamma_{F \triangle G}\right)=\tau\left(\rho_{E, F}, \rho_{F, G}\right),
\end{aligned}
$$

which means that $\rho$ is a pseudo-metric with respect to the triangle function $\tau$.

Remark 4.7. Since $E \triangle G=(E \triangle F) \triangle(F \triangle G)$, then it is easy to observe that $\rho$ is translation invariant, i.e., $\rho_{E, G}=\gamma_{E \triangle G}=\gamma_{(E \triangle F) \triangle(F \triangle G)}=\rho_{E \triangle F, F \triangle G}$.

For a fixed triangle function $\tau$ denote by $\Gamma_{\tau}(\Sigma, \gamma)$ the set of all pseudo-metrics $\rho$ generated by $\tau$-decomposable antimonotone submeasures $\gamma$ on $\Sigma$, i.e., $\rho_{E, F}=\gamma_{E \triangle F}$ with $E, F \in \Sigma$. Define a relation $\preceq$ on $\Gamma_{\tau}(\Sigma, \gamma)$ as follows

$$
\rho \preceq \varrho \Leftrightarrow \rho_{E, F} \geq \varrho_{E, F} \text { for each } E, F \in \Sigma \text {. }
$$

Clearly, $\preceq$ is a partial order on $\Gamma_{\tau}(\Sigma, \gamma)$ and $\nu_{E, F}=\varepsilon_{0}$ for each $E, F \in \Sigma$ is an element such that $\nu \preceq \rho$ for every $\rho \in \Gamma_{\tau}(\Sigma, \gamma)$. 
Let $\vartheta$ be a triangle function on $\Delta^{+}$such that $\vartheta \gg \tau$. Define a binary operation $\oplus_{\vartheta}$ on $\Gamma_{\tau}(\Sigma, \gamma)$ such that

$$
(\rho \oplus \vartheta \varrho)_{E, F}:=\vartheta\left(\rho_{E, F}, \varrho_{E, F}\right), \quad E, F \in \Sigma,
$$

for all $\rho, \varrho \in \Gamma_{\tau}(\Sigma, \gamma)$. We show that $\rho \oplus_{\vartheta} \varrho \in \Gamma_{\tau}(\Sigma, \gamma)$. Indeed, for an arbitrary $E \in \Sigma$ we have

$$
\left(\rho \oplus_{\vartheta} \varrho\right)_{E, E}=\vartheta\left(\rho_{E, E}, \varrho_{E, E}\right)=\vartheta\left(\varepsilon_{0}, \varepsilon_{0}\right)=\varepsilon_{0}
$$

and the symmetry $\left(\rho \oplus_{\vartheta} \varrho\right)_{E, F}=\left(\rho \oplus_{\vartheta} \varrho\right)_{F, E}$ is obvious for each $E, F \in \Sigma$. Also, by triangle inequality (10) and domination of $\vartheta$ over $\tau$ we have

$$
\begin{aligned}
\left(\rho \oplus_{\vartheta} \varrho\right)_{E, G} & =\vartheta\left(\rho_{E, G}, \varrho_{E, G}\right) \geq \vartheta\left(\tau\left(\rho_{E, F}, \rho_{F, G}\right), \tau\left(\varrho_{E, F}, \varrho_{F, G}\right)\right) \\
& \geq \tau\left(\vartheta\left(\rho_{E, F}, \varrho_{E, F}\right), \vartheta\left(\rho_{F, G}, \varrho_{F, G}\right)\right) \\
& =\tau\left(\left(\rho \oplus_{\vartheta} \varrho\right)_{E, F},\left(\rho \oplus_{\vartheta} \varrho_{F, G}\right),\right.
\end{aligned}
$$

where $E, F, G \in \Sigma$. Thus $\rho \oplus_{\vartheta} \varrho$ is a pseudo-metric on $\Sigma$. Note that for all $\rho \in \Gamma_{\tau}(\Sigma, \gamma)$ and $\vartheta \gg \tau$ it holds

$$
\left(\nu \oplus_{\vartheta} \rho\right)_{E, F}=\vartheta\left(\nu_{E, F}, \rho_{E, F}\right)=\vartheta\left(\varepsilon_{0}, \rho_{E, F}\right)=\rho_{E, F}
$$

for each $E, F \in \Sigma$. The operation $\oplus_{\vartheta}$ is clearly commutative and associative. Thus we have the following result.

Proposition 4.8. Let $\vartheta \gg \tau$. The triple $\left(\Gamma_{\tau}(\Sigma, \gamma), \oplus_{\vartheta}, \preceq\right)$ is a partially ordered commutative semigroup with the neutral element $\nu$.

A relationship between the relation $\preceq$ and the operation $\oplus_{\vartheta}$ is described in the following theorem.

Theorem 4.9. Let $\vartheta \gg \tau$. Then for all $\rho, \varrho, \sigma \in \Gamma_{\tau}(\Sigma, \gamma)$ the relationship $\rho \oplus_{\vartheta} \sigma \preceq \varrho \oplus_{\vartheta} \sigma$ holds whenever $\rho \preceq \varrho$.

Proof. The relation $\rho \preceq \varrho$ means that $\rho_{E, F} \geq \varrho_{E, F}$ for each $E, F \in \Sigma$. From monotonicity of $\vartheta$ we have $\vartheta\left(\rho_{E, F}, \sigma_{E, F}\right) \geq \vartheta\left(\varrho_{E, F}, \sigma_{E, F}\right)$ and the proof is complete.

Recall that $\Pi_{M}$ (the pointwise defined operation on $\Delta^{+}$given by (8) with the minimum t-norm $M$ ) dominates each triangle function $\tau$, cf. 21, Proposition 6.2]. Also, a semilattice is an idempotent commutative semigroup. A semilattice is bounded if it includes the neutral element.

Theorem 4.10. The ordered pair $\left(\Gamma_{\tau}(\Sigma, \gamma), \oplus_{\Pi_{M}}\right)$ is a bounded semilattice with the properties

(i) $\rho \preceq \varrho$ if and only if $\rho \oplus_{\Pi_{M}} \varrho=\varrho$;

(ii) $\left(\sigma \oplus_{\vartheta} \rho\right) \oplus_{\Pi_{M}}\left(\sigma \oplus_{\vartheta} \varrho\right) \preceq \sigma \oplus_{\vartheta}\left(\rho \oplus_{\Pi_{M}} \varrho\right)$

for each $\rho, \varrho, \sigma \in \Gamma_{\tau}(\Sigma, \gamma)$ provided that $\vartheta \gg \tau$.

Proof. Since $\nu \in \Gamma_{\tau}(\Sigma, \gamma)$ is the neutral element, then $\left(\Gamma_{\tau}(\Sigma, \gamma), \oplus_{\Pi_{M}}\right)$ is a bounded semilattice.

(i) If $\rho \preceq \varrho$, then $\rho_{E, F} \geq \varrho_{E, F}$ for each $E, F \in \Sigma$, thus $\rho \oplus_{\Pi_{M}} \varrho=\varrho$. The opposite statement is obvious.

(ii) For the proof of second part it is enough to observe that $\rho \preceq \rho \oplus_{\Pi_{M}} \varrho$ and $\varrho \preceq \rho \oplus_{\Pi_{M}} \varrho$. By Theorem 4.9 we have $\sigma \oplus_{\vartheta} \rho \preceq \sigma \oplus_{\vartheta}\left(\rho \oplus_{\Pi_{M}} \varrho\right)$ and $\sigma \oplus_{\vartheta} \varrho \preceq \sigma \oplus_{\vartheta}\left(\rho \oplus_{\Pi_{M}} \varrho\right)$. Since $\left(\Gamma_{\tau}(\Sigma, \gamma), \oplus_{\Pi_{M}}\right)$ is a semilattice, the property (ii) follows. 


\section{Decomposable measures and the probabilistic Haus- dorff distance}

As it was already mentioned in this paper, Shen introduced in 23. a class of probabilistic decomposable measures w.r.t. the triangle function $\Pi_{\top}$ given by (8). Now we extend his certain results related to the probabilistic Hausdorff distance. For that reason we recall necessary notions from [23].

Let $(\Omega, \mathscr{F}, \tau)$ be a PM-space.

(i) The probabilistic diameter of a non-empty subset $E$ of $\Omega$ is a mapping $D_{E}:[0,+\infty) \rightarrow[0,1]$ defined by

$$
D_{E}(t):=\sup _{s<t} \inf _{p, q \in E} H_{p, q}(s) .
$$

(ii) A set $E$ is said to be probabilistic bounded if $\sup _{t} D_{E}(t)=1$. The collection of all probabilistic bounded subsets of $\Omega$ will be denoted by $P B(\Omega)$.

(iii) Let $p \in \Omega$ and $F \in P B(\Omega)$. The probabilistic distance from $p$ to $F$ is defined as

$$
d_{p, F}(t):= \begin{cases}0, & t=0, \\ \sup _{s<t} \sup _{q \in F} H_{p, q}(s), & t \in \mathbb{R}_{+},\end{cases}
$$

with the convention $d_{p, \emptyset}=1-\varepsilon_{0}$.

(iv) Given $E, F \in P B(\Omega)$, the probabilistic distance from $E$ to $F$ is defined as

$$
d_{E, F}(t):= \begin{cases}0, & t=0 \\ \sup _{s<t} \inf _{p \in E} \sup _{q \in F} H_{p, q}(s), & t \in \mathbb{R}_{+},\end{cases}
$$

with the convention $d_{\emptyset, F}=\varepsilon_{0}$.

Definition 5.1. Let $(\Omega, \mathscr{F}, \tau)$ be a PM-space and $E, F \in P B(\Omega)$. The probabilistic Hausdorff distance between $E$ and $F$ is a mapping $\mathscr{H}_{E, F}:[0,+\infty) \rightarrow[0,1]$ defined by

$$
\mathscr{H}_{E, F}(t):= \begin{cases}0, & t=0, \\ \sup _{s<t} M\left(\inf _{p \in E} \sup _{q \in F} H_{p, q}(s), \inf _{q \in F} \sup _{p \in E} H_{p, q}(s)\right), & t \in \mathbb{R}_{+} .\end{cases}
$$

From now on let $\Sigma:=P(\Omega)$ be the power set of $\Omega$. Using the probabilistic Hausdorff distance we may define a mapping $\Lambda^{\mathscr{H}}: \Sigma \rightarrow \Delta^{+}$by

$$
\Lambda_{X}^{\mathscr{H}}:=\mathscr{H}_{E^{c}, \Omega},
$$

where $E^{c}$ is the complement of a set $E \in \Sigma$.

Theorem 5.2. Let $(\Omega, \mathscr{F}, \tau)$ be a PM-space. Then $\Lambda^{\mathscr{H}}$ is an antimonotone set function with $\Lambda_{\emptyset}^{\mathscr{H}}=\varepsilon_{0}$ and

$$
\Lambda_{E \cup F}^{\mathscr{H}} \leq \Pi_{M}\left(\Lambda_{E}^{\mathscr{H}}, \Lambda_{F}^{\mathscr{H}}\right), \quad E, F \in \Sigma .
$$

Proof. From definitions above it is obvious that $\Lambda_{\emptyset}^{\mathscr{H}}=\varepsilon_{0}$. Also, for $E, F \in \Sigma$ such that $E \subseteq F$ we have $F^{c} \subseteq E^{c}$, and therefore

$$
\Lambda_{E}^{\mathscr{H}}(t)=\mathscr{H}_{E^{c}, \Omega}(t)=\sup _{s<t} \inf _{q \in \Omega} \sup _{p \in E^{c}} H_{p, q}(s) \geq \sup _{s<t} \inf _{q \in \Omega} \sup _{p \in F^{c}} H_{p, q}(s)=\mathscr{H}_{F^{c}, \Omega}(t)=\Lambda_{F}^{\mathscr{H}}(t)
$$

for each $t \geq 0$, which proves antimonotonicity of $\Lambda^{\mathscr{H}}$.

Finally, the inclusions $E \subseteq E \cup F, F \subseteq E \cup F$ and antimonotonicity of $\Lambda^{\mathscr{H}}$ yields

$$
\Lambda_{E \cup F}^{\mathscr{H}}(t) \leq M\left(\Lambda_{E}^{\mathscr{H}}(t), \Lambda_{F}^{\mathscr{H}}(t)\right)=\Pi_{M}\left(\Lambda_{E}^{\mathscr{H}}(t), \Lambda_{F}^{\mathscr{H}}(t)\right)
$$

for each $t \geq 0$, i.e., $\Lambda_{E \cup F}^{\mathscr{H}} \leq \Pi_{M}\left(\Lambda_{E}^{\mathscr{H}}, \Lambda_{F}^{\mathscr{H}}\right)$. 
Remark 5.3. Equivalently, we may say that $\Lambda^{\mathscr{H}}$ is a probabilistic-valued antimonotone $\Pi_{M}$ decomposable supermeasure on $\Sigma\left(\Pi_{M}\right.$-probabilistic submeasure in the terminology of Shen [23]). Under certain condition on the domain of $\Lambda^{\mathscr{H}}$ we will be able to show that it is a $\tau$-decomposable measure w.r.t. an arbitrary triangle function $\tau$ (thus, also for $\Pi_{M}$ ), see Theorem 5.6 below.

Definition 5.4. A set $E \in \Sigma$ is said to be $\Lambda^{\mathscr{H}}$-measurable w.r.t. a triangle function $\tau$, if for each $G \in \Sigma$ it holds

$$
\Lambda_{G}^{\mathscr{H}}=\tau\left(\Lambda_{G \cap E}^{\mathscr{H}}, \Lambda_{G \cap E^{c}}^{\mathscr{H}}\right) .
$$

Theorem 5.5. Let $(\Omega, \mathscr{F}, \tau)$ be a PM-space. Then each class

$$
\mathbb{S}_{\tau}=\left\{E \in \Sigma ; E \text { is } \Lambda^{\mathscr{H}} \text {-measurable w.r.t. } \tau\right\}
$$

is an algebra.

Proof. Since $\mathscr{H}_{\emptyset, \Omega}=\mathscr{H}_{\Omega, \Omega}=\varepsilon_{0}$, then $\emptyset, \Omega \in \mathbb{S}_{\tau}$. Moreover, from definition it follows that if $E \in \mathbb{S}_{\tau}$, then $E^{c} \in \mathbb{S}_{\tau}$ (and vice versa). Thus, we only have to prove that $\mathbb{S}_{\tau}$ is closed under the formation of union.

Let $E, F \in \mathbb{S}_{\tau}$ and consider an arbitrary $G \in \Sigma$. Since $F \in \mathbb{S}_{\tau}$, then

$$
\Lambda_{G \cap(E \cup F)}^{\mathscr{H}}=\tau\left(\Lambda_{G \cap(E \cup F) \cap F}^{\mathscr{H}}, \Lambda_{G \cap(E \cup F) \cap F^{c}}^{\mathscr{H}}\right)=\tau\left(\Lambda_{G \cap F}^{\mathscr{H}}, \Lambda_{G \cap E \cap F^{c}}^{\mathscr{H}}\right),
$$

and therefore we get

$$
\begin{aligned}
\tau\left(\Lambda_{G \cap(E \cup F)}^{\mathscr{H}}, \Lambda_{G \cap(E \cup F)^{c}}^{\mathscr{H}}\right) & =\tau\left(\tau\left(\Lambda_{G \cap F}^{\mathscr{H}}, \Lambda_{G \cap F^{c} \cap E}^{\mathscr{H}}\right), \Lambda_{G \cap(E \cup F)^{c}}^{\mathscr{H}}\right) \\
& =\tau\left(\tau\left(\Lambda_{G \cap F}^{\mathscr{H}}, \Lambda_{G \cap F^{c} \cap E}^{\mathscr{H}}\right), \Lambda_{G \cap F^{c} \cap E^{c}}^{\mathscr{H}}\right) \\
& =\tau\left(\Lambda_{G \cap F}^{\mathscr{H}}, \tau\left(\Lambda_{G \cap F^{c} \cap E}^{\mathscr{H}}, \Lambda_{G \cap F^{c} \cap E^{c}}^{\mathscr{H}}\right)\right) \\
& =\tau\left(\Lambda_{G \cap F}^{\mathscr{H}}, \Lambda_{G \cap F^{c}}^{\mathscr{H}}\right) \\
& =\Lambda_{G}^{\mathscr{H}} .
\end{aligned}
$$

Thus, the set $E \cup F$ is $\Lambda^{\mathscr{H}}$-measurable w.r.t. $\tau$, i.e., $E \cup F \in \mathbb{S}_{\tau}$.

Theorem 5.6. Let $(\Omega, \mathscr{F}, \tau)$ be a PM-space. The restriction of the set function $\Lambda^{\mathscr{H}}$ to $\mathbb{S}_{\tau}$ is a $\tau$-decomposable measure.

Proof. Let $E, F \in \mathbb{S}_{\tau}$ be disjoint. Since $E \cup F$ is $\Lambda^{\mathscr{H}}$-measurable w.r.t. $\tau$, then

$$
\Lambda_{E \cup F}^{\mathscr{H}}=\tau\left(\Lambda_{(E \cup F) \cap E}^{\mathscr{H}}, \Lambda_{(E \cup F) \cap E^{c}}^{\mathscr{H}}\right)=\tau\left(\Lambda_{E}^{\mathscr{H}}, \Lambda_{F}^{\mathscr{H}}\right),
$$

i.e., $\Lambda^{\mathscr{H}}$ is a $\tau$-decomposable measure on $\mathbb{S}_{\tau}$.

\section{Concluding remarks}

In this paper we have introduced a generalized decomposable (sub)measure notion related to triangle functions in probabilistic metric spaces which covers our previously introduced notions of (sub)measures as well as certain recent notions from the literature. We have given a characterization of decomposable measures and discussed construction methods providing new decomposable (sub)measures from the given ones. In connection with this "aggregation" the following problem arises:

Problem 5.7. Characterize all the mappings which preserve the class of $\tau$-decomposable measures.

Further, we have extended Shen's results related to probabilistic-valued decomposable measures and the probabilistic Hausdorff distance for an arbitrary triangle function. We have proved that the class of all measurable sets forms an algebra on its power set. Thus, the second problem repeats the Shen's question in [23]: 
Problem 5.8. Is the class $\mathbb{S}_{\tau}$ of all $\Lambda^{\mathscr{H}}$-measurable sets w.r.t. a triangle function $\tau$ a $\sigma$ algebra? Does this property depend on a choice of triangle function $\tau$ ?

As it is well-known, triangle functions have been used and discussed almost exclusively in the theory of probabilistic metric and probabilistic normed spaces. Particular examples of triangle functions also appear in, e.g., the treatment of fuzzy numbers or in information theory (compare also [17]). As it was described in the introduction the probabilistic-valued (sub)measures w.r.t. triangle function $\tau_{M}$ may be naturally interpreted in the context of fuzzy sets and fuzzy numbers. Thus, one can be interested in the following:

Problem 5.9. Is there room in these theories (both PM-spaces as well as fuzzy sets) for $\tau$ decomposable (sub)measures w.r.t. triangle functions of other type? Which interpretation do this objects have?

Furthermore, the idea of probabilistic integral introduced in [2] motivates to develop an integral w.r.t. probabilistic-valued decomposable (sub)measures for an arbitrary triangle function. This is already done in our recent paper 7]. This approach may be understood as a modification of Aumann integral, or, of Choquet-type integral based on interval-valued measures as discussed e.g. in [12].

\section{Acknowledgement}

We are grateful to the referees for their suggestions which essentially improved previous version of the manuscript. We acknowledge a partial support of grants VEGA 1/0171/12, VVGS-PF2013-115 and VVGS-2013-121.

\section{References}

[1] De Baets, B., Mesiar, R.: T-partitions. Fuzzy Sets and Systems 97 (1998), 211-223.

[2] Bahrami, F., Mohammadbaghban, M.: Probabilistic $L^{p}$ spaces. J. Math. Anal. Appl. 402(2) (2013), 505-517.

[3] Dubois, D. Kerre, E. E., Mesiar, R. Prade, H.: Fuzzy interval analysis. In: Fundamentals of Fuzzy Sets, D. Dubois and H. Prade (Eds.), Kluwer, Dordrecht, 2000, pp. $483-584$.

[4] Gilio, A, Sanfilippo, G.: Quasi conjunction, quasi disjunction, t-norms and t-conorms: probabilistic aspects. Inf. Sci. 245 (2013), 146-167.

[5] Grabisch, M., Pap, E., Mesiar, R., Marichal, J.-L.: Aggregation Functions. Cambridge Univ. Press, New York, 2009.

[6] Grzymala-Busse, J. W., Clark, P. G., Kuehnhausen, M.: Generalized probabilistic approximations of incomplete data. Internat. J. Approx. Reason. 55 (2014), 180-196.

[7] Halčinová, L., Hutník: An integral with respect to probabilistic-valued decomposable measures. (submitted).

[8] Halčinová, L., Hutník, O., Mesiar, R.: On distance distribution functions-valued submeasures related to aggregation functions. Fuzzy Sets and Systems 194(1) (2012), 1530 .

[9] Halčinová, L., Hutník, O., Mesiar, R.: On some classes of distance distribution functions-valued submeasures. Nonlinear Anal. 74(5) (2011), 1545-1554.

[10] Hunter, A.: A probabilistic approach to modelling uncertain logical arguments. Internat. J. Approx. Reason. 54 (2013), 47-81. 
[11] Hutník, O., Mesiar, R.: On a certain class of submeasures based on triangular norms. Internat. J. Uncertain. Fuzziness Knowledge-Based Systems 17 (2009), 297-316.

[12] JANG, L.-CH.: A note on convergence properties of interval-valued capacity functionals and Choquet integrals. Inf. Sci. 183(1) (2012), 151-158.

[13] KAMpé DE FÉRIET, J.-M.: La théorie general de l'information et la mesure subjective de l'information. In: Lecture Notes in Math. 398, Springer-Verlag, Heidelberg 1974, pp. 1-35.

[14] Klement, E. P., Mesiar, R., Pap, E.: Triangular Norms. Trends in Logic, Studia Logica Library 8, Kluwer Academic Publishers, 2000.

[15] Menger, K.: Statistical metrics. Proc. Nat. Acad. Sci. U.S.A. 28 (1942), 535-537.

[16] Moore, R. E., Bierbaum, F.: Methods and Applications of Interval Analysis. Studies in Applied and Numerical Mathematics, SIAM, Philadelphia, 1979.

[17] PAP, E., ŠTajner, I.: Generalized pseudo-convolution in the theory of probabilistic metric spaces, information, fuzzy numbers, optimization, system theory. Fuzzy Sets and Systems 102 (1999), 393-415.

[18] Pradera, A., Trillas, E.: A note on pseudometrics aggregation, Int. J. Gen. Syst. 31 (2002), 41-51.

[19] Saminger, S., Mesiar, R., Bodenhofer, U.: Domination of aggregation operators and preservation of transitivity. Internat. J. Uncertain. Fuzziness Knowledge-Based Systems 10 (2002), 11-35.

[20] Saminger-Platz, S. Sempi, C.: A primer on triangle functions I., Aequationes Math. 76(3) (2008), 201-240.

[21] Saminger-Platz, S. Sempi, C.: A primer on triangle functions II., Aequationes Math. 80(3) (2010), 239-268.

[22] Schweizer, B., Sklar, A.: Probabilistic Metric Spaces. North-Holland Publishing, New York, 1983.

[23] SHEn, Y.: On probabilistic Hausdorff distance and a class of probabilistic decomposable measures. Inf. Sci. 263 (2014), 126-140.

[24] Sugeno, M., Murofushi, T.: Pseudo-additive measures and integrals. J. Math. Anal. Appl. 122 (1987), 197-222.

[25] Yu, Z., Li, L. Wong, H.-S., You, J., Han, G., GaO, Y., Yu, G.: Probabilistic cluster structure ensemble. Inf. Sci., http://dx.doi.org/10.1016/j.ins.2014.01.030.

Lenka Halčinová, Ondrej Hutník, Jana Molnárová

Institute of Mathematics, Faculty of Science, Pavol Jozef Šafárik University in Košice,

Current address: Jesenná 5, SK 04001 Košice, Slovakia,

E-mail addresses: lenka.halcinova@student.upjs.sk

ondrej.hutnik@upjs.sk

jana.molnarova@student.upjs.sk 